\title{
Prehypertension in dyslipidemic individuals; relationship to metabolic parameters and intima-media thickness
}

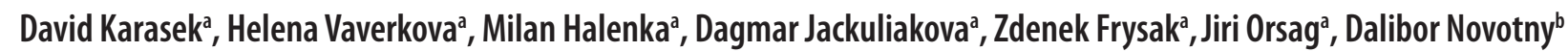

Introduction. Like hypertension, prehypertension is associated with cardiovascular disease.

Aims. The aim of this study was to evaluate: a) the prevalence of prehypertension/hypertension in individuals with various dyslipidemic phenotypes; $b$ ) the relation between blood pressure (BP) and other risk factors for atherosclerosis; c) atherogenic potential of prehypertension by the assessment of intima-media thickness of the arteria carotis communis (IMT).

Methods. 667 clinically asymptomatic subjects were divided into four dyslipidemic phenotypes (DLP) according to apolipoprotein B (apoB) and triglycerides (TG): DLP1 ( $n=198$, normo-apoB/normo-TG), DLP2 ( $n=179$, normo-apoB/ hyper-TG), DLP3 ( $n=87$, hyper-apoB/normo-TG), DLP4 ( $n=203$, hyper-apoB/hyper-TG). DLP1 served as a control group. Results. There was significantly higher prevalence of prehypertension and hypertension in subjects with dyslipidemia (DLP2 43.0\%, 41.3\%; DLP3 42.5\%, 29.9\%; DLP4 42.4\%, 47.8\%) than in normolipidemic individuals (DLP1 32.8\%, 20.2\%). Systolic and diastolic blood pressure (SBP + DBP) correlated with age, total cholesterol, TG, non-HDL-cholesterol, body mass index and waist circumference; SBP additionally with C-peptide, fasting glycemia; DBP additionally with apoB, homeostasis model assessment (HOMA) and plasminogen activator inhibitor-1. The IMT of hypertensive and of prehypertensive subjects was higher than that of subjects with normal BP in all DLPs.

Conclusions. The prevalence of prehypertension was higher in all dyslipidemic patients. The common prevalence of prehypertension/hypertension was highest in the hypertriglyceridemic subjects. Prehypertensive and hypertensive patients had higher IMT than normotensive individuals in all DLPS.

Key words: prehypertension, hypertension, dyslipidemia, insulin resistance, intima-media thickness

Received: October 3, 2011; Accepted with revision: April 12, 2012; Available online: June 1, 2012 http://dx.doi.org/10.5507/bp.2012.046

${ }^{a}$ Department of Internal Medicine III - Nephrology, Rheumatology and Endocrinology, University Hospital Olomouc, Czech Republic ${ }^{b}$ Department of Biochemistry, University Hospital Olomouc

Corresponding author: David Karasek, e-mail:david.karasek@fnol.cz

\section{INTRODUCTION}

Prehypertension has been defined as systolic blood pressure (SBP) 120 to 139 or diastolic blood pressure (DBP) 80 to $89 \mathrm{~mm} \mathrm{Hg}$, based on two or more properly measured seated blood pressure $(\mathrm{BP})$ readings on each of two or more office visits ${ }^{1}$. Prehypertension tends to increase in severity over time ${ }^{2}$ and associates with increased incidence of cardiovascular disease (CVD), particularly in those with BP levels in the 130 to $139 / 85$ to $89 \mathrm{~mm} \mathrm{Hg}$ range $^{3,4}$. Compared with normal BP, prehypertension has been associated with a $27 \%$ increase in all-cause and $66 \%$ increase in CVD mortality ${ }^{5}$. A study of 1999 to 2000 NHANES data has suggested that $64 \%$ of prehypertensive subjects have $\geq 1$ other abnormal CVD risk factors, the percentage increased to $94 \%$ in those $\geq 60$ years of age ${ }^{6}$. The risk ratios for obesity, dyslipidemia, metabolic syndrome, and diabetes are all greater in prehypertensive than normotensive subjects ${ }^{7,8}$. The dyslipidemias represent heterogenic metabolic disorders. Sniderman suggested an algorithm of dyslipidemia classification based on apolipoprotein B (apoB) and triglyceride (TG) concentrations ${ }^{9}$. The classification divides dyslipidemias into four dyslipidemic phenotypes (DLP) on the basis of apoB and TG concentrations: DLP 1 (normo-apoB / normo-TG), DLP 2 (normo-apoB / hyper-TG), DLP 3 (hyper-apoB / normo-TG) and DLP 4 (hyper-apoB / hyper-TG). Assessment of ApoB used instead of LDL cholesterol (LDL-C) in this diagnostic algorithm is rational and fully justified. ApoB is a better predictor for the risk of coronary artery disease than total cholesterol (TC), LDL-C and non-HDL cholesterol (non-HDL-C). This has repeatedly been shown in a large number of prospective epidemiological studies dealing with lipids and apoB (ref. ${ }^{10}$ ).

The aims of the present study were to investigate: the prevalence of prehypertension and hypertension in dyslipidemic individuals without clinical manifestation of atherosclerosis; the relation between blood pressure and other risk factors for atherosclerosis-and the atherogenic capacity of prehypertension by assessment of the intima-media thickness of the arteria carotis communis (IMT), as a surrogate marker of subclinical atherosclerosis manifestation. 


\section{MATERIALS AND METHODS}

\section{Study design and subjects}

The study was carried out as a cross-sectional study on subjects who had been examined at the Lipid Center of the $3^{\text {rd }}$ Department of Internal Medicine, University Hospital Olomouc, Czech Republic in the period October 2005 to June 2011. All subjects (patients with dyslipidemia, their spouses, relatives and healthy volunteers; $\mathrm{n}=828$ ) filled out a questionnaire on their medical history, especially cardiovascular status, medication and smoking habits. All subjects were tested for an underlying cause of secondary hyperlipidemia: diabetes mellitus, hypothyroidism, hepatic or renal impairment and nephrotic syndrome. Subjects with these diagnoses were not enrolled in the study. Other exclusion criteria were history of clinically manifest atherosclerosis (coronary artery disease, cerebrovascular ischemic disease and peripheral arterial disease), hypolipidemic treatment in the previous 8 weeks, hormone therapy with estrogens and clinical presence of acute infectious disease or trauma. The study was reviewed and approved by the institutional Ethics Committee of the Faculty of Medicine and Dentistry and University Hospital and informed consent was obtained from all participants. Individuals who met the above mentioned criteria ( $\mathrm{n}=667)$, were divided into four dyslipidemic phenotypes (DLP) based on apolipoprotein B and triglycerides: DLP $1(\mathrm{n}=198$, apoB $<1.2$ $\mathrm{g} / \mathrm{L}$ and $\mathrm{TG}<1.5 \mathrm{mmol} / \mathrm{L})$, DLP $2(\mathrm{n}=179$, apoB $<1.2 \mathrm{~g} / \mathrm{L}$ and $\mathrm{TG} \geq 1.5 \mathrm{mmol} / \mathrm{L}), \mathrm{DLP} 3(\mathrm{n}=87, \mathrm{apoB} \geq 1.2 \mathrm{~g} / \mathrm{L}$ and $\mathrm{TG}<1.5 \mathrm{mmol} / \mathrm{L})$, DLP $4(\mathrm{n}=203$, apoB $\geq 1.2 \mathrm{~g} / \mathrm{L}$ and $\mathrm{TG} \geq 1.5 \mathrm{mmol} / \mathrm{L}$ ). The use of TG value in this algorithm is justified as well. It had been proved that the amount of small dense LDL increases with TG concentration 1.5 $\mathrm{mmol} / \mathrm{L}\left(\right.$ ref. ${ }^{9}{ }^{11}$ ). A value $1.2 \mathrm{~g} / \mathrm{L}$ for apoB was chosen, because at this level the cardiovascular risk rapidly increased ${ }^{9,12}$. This value corresponds to the $75^{\text {th }}$ percentile of the North American population, the level chosen to define an elevated LDL-C (ref. $\left.{ }^{13,14}\right)$. Subjects with DLP 1 - who were normolipidemic (normo-apoB / normo-TG) - served as a control group. The presence of metabolic syndrome was assessed according to National Cholesterol Education Program Adult Panel III (ref. ${ }^{15}$ ). The ten-year risk of having a heart attack using risk assessment tool based on information from Framingham Heart Study was estimated in all participants ${ }^{16}$.

\section{Blood pressure measurement}

Body mass index (BMI), waist circumference, SBP and DBP were determined. The auscultatory method of BP measurement with a properly calibrated and validated mercury sphygmomanometer was used. Persons were seated quietly for at least $5 \mathrm{~min}$ in a chair, with feet on the floor, and arm supported at heart level. An appropriate-sized cuff was used to ensure accuracy. The first and fifth Korotkoff sounds were used to identify SBP and DBP (ref. ${ }^{1}$ ). At least three sitting BP measurement with a 30-second interval were taken and the mean from the last two was calculated. Subjects with $\mathrm{SBP} \geq 120$ and
$<140$ or DBP $\geq 80$ and $<90 \mathrm{mmHg}$ were defined as prehypertensive. Patients treated by antihypertensive drugs, or with $\mathrm{SBP} \geq 140$ or $\mathrm{DBP} \geq 90 \mathrm{mmHg}$ were taken as hypertensive ${ }^{1}$. There was a substantial part of treated patients within the hypertensive group. Hypertensive patients with DLP $1(\mathrm{n}=40)$ were treated by ACE inhibitors (ACE-I) or angiotensin II receptor blockers (ARBs) in $37.5 \%$, by calcium channel blockers $(\mathrm{CaCBs})$ in $5.0 \%$, by beta blockers (BBs) in $35.0 \%$, and by diuretics in $12.5 \%$. Patients with hypertension and DLP $2(n=74)$ were treated by ACE-I or ARBs in $48.6 \%$, by CaCBs in $18.9 \%$, by $\mathrm{BBs}$ in $36.5 \%$, by diuretics in $18.9 \%$, and by imidazoline- 1 receptor ligands (IRLs) in $9.5 \%$; one patient was treated by alfa-metyldopa. Hypertensive patients $(n=26)$ with DLP 3 were treated by ACE-I or ARBs in 26.9\%, by $\mathrm{CaCBs}$ in $11.5 \%$, and by $\mathrm{BBs}$ in $11.5 \%$. Patients with hypertension and DLP $4(n=97)$ were treated by ACE-I or ARBs in $50.5 \%$, by CaCBs in $17.5 \%$, by BBs in $35.0 \%$, by diuretics in $20.6 \%$, and by IRLs in $4.1 \%$; one patient was treated by doxazosine.

\section{Biochemical analyses}

Venous blood samples were drawn in the morning after a $12 \mathrm{~h}$ fast. Total cholesterol, triglycerides and HDLcholesterol were determined enzymatically on Modular SWA analyzer (Roche, Basel, Switzerland) using commercially available kits (Cholesterol SYS 917, Triglycerides GPO-PAP and HDL cholesterol plus, $3^{\text {rd }}$ generation kits, Roche, Basel, Switzerland). Determination of HDL-C was made by a direct method without precipitation of apo B containing lipoproteins. LDL-C levels were calculated according to the Friedewald formula (for TG < $4.5 \mathrm{mmol} / \mathrm{L})$. We also calculated non-HDL cholesterol (non-HDL-C = TC - HDL-C). Concentration of apoB and apolipoprotein A1 (apoA1) was determined immunoturbidimetrically on Modular SWA analyzer (TinaQuant Apo A1, TinaQuant Apo B kits, all Roche, Basel, Switzerland). High-sensitivity C-reactive protein (hs-CRP) was assessed by means of an ultra sensitive latex immunoturbidimetric method on Modular SWA analyzer (CRP latex TinaQuant kit, all Roche, Basel, Switzerland). Glycemia was determined by enzymatic-colorimetric method (Glucose GOD-PAP kit) on Modular SWA analyzer. Insulin was determined using the commercially available kits - Insuline (Immunotech, Marseille, France) using specific antibodies by IRMA (immunoradiometric assay) method. The result obtained was then used for calculation of the parameter of insulin resistance HOMA [homeostasis model assessment, described by Matthews et al.: fasting glycemia (mmol/L) ${ }_{*}$ fasting insulin (mU/L) / 22.5] ( ref. $\left.^{17}\right)$. C-peptide and intact proinsulin were determined using the commercially available kits - C-peptide (Immunotech, Marseille, France) and Proinsulin (DRG Instruments $\mathrm{GmbH}$, Marburg, Germany) using specific antibodies by IRMA method for C-peptide and radioimmune assay (RIA) method for proinsulin. Concentrations of insulin, proinsulin, C-peptide and hs-CRP were measured in the serum stored at $-80^{\circ} \mathrm{C}$. Serum levels of the soluble intercellular cell adhesion molecule-1 (s-ICAM-1) and plas- 
minogen activator inhibitor-1 (PAI-1) were measured by enzyme-linked immunosorbent assay (ELISA) using commercially available standard kits s-ICAM-1 (Immunotech, Marseille, France) and PAI-1 (Technoclone, Vienna, Austria). These markers of endothelial damage were used because we previously found significant differences in their levels between individual DLPs ( ref. $^{18,19}$ ).

\section{IMT measurement}

Ultrasound scanning of IMT was performed with a $10 \mathrm{MHz}$ linear array transducer (Philips Sonos 5500, 2004). All measurements were performed with subjects in a supine position. The head was tilted to the one side at an angle of $45^{\circ}$. The longitudinal B-mode image of the common carotid artery (CCA) was displayed just before the widening of the bulb. When an optimal longitudinal image of the far wall of the CCA in the region of $1 \mathrm{~cm}$ proximally from the bulb was obtained, it was frozen on the $\mathrm{R}$ wave according to a simultaneous ECG and videotaped. Three video records were made on both CCA. IMT measurements were processed off-line using the software Image-Pro Plus (Version 4.0, Media-Cybernetics, Silver Spring, USA). The region under evaluation was the CCA wall $1-2 \mathrm{~cm}$ distant proximally from the mentioned border. The average of all mean IMT of three frozen images of both sides was chosen as the outcome variable. The measurement of IMT was made without knowledge of laboratory results.

\section{Statistical analyses}

All values are expressed as means $\pm \mathrm{SD}$ or as median with 25 and 75 percentiles for variables with non-normal distribution. The Kolmogorov-Smirnov test was used to test for normal distribution. Variables with non-normal distribution (TG, proinsulin, insulin, C-peptide, HOMA, hs-CRP, PAI-1) were log transformed to normalize their distribution before statistical analysis. Differences in means between groups were analyzed using ANOVA after adjustment for age, sex, BMI, and smoking. Differences in categorical variables were analyzed by $\mathrm{X}^{2}$ test. For statistical evaluation of correlations between parameters we used a univariate Pearson correlation analysis. Multivariate regression analysis was used for testing for an independent association between dependent and independent variables. Statistical analysis was performed using SPSS for Windows version 12.0 (Chicago, Illinois, USA). Probability values of $P<0.05$ were considered as statistically significant.

\section{RESULTS}

\section{Basic characteristics}

Table 1 shows the biochemical and anthropometric characteristics in individual DLPs. Individuals with hyperapoB DLPs (DLP 3 and 4) had significantly increased total cholesterol and LDL-C; individuals with hyper-TG DLPs (DLP 2 and 4) had decreased HDL-C and apoA1 compared to normolipidemic subjects (DLP 1). NonHDL-C and apoB/apoA1 significantly rose from DLP 1 to DLP 4. Subjects with hyper-TG DLPs (DLP 2 and 4) showed increased markers of insulin resistance (HOMA, C-peptide, insulin, proinsulin), obesity (BMI, waist circumference) and also increased prevalence of metabolic syndrome. They had higher blood pressure (SBP, DBP) and levels of PAI-1. Patient with DLP4 had significantly increased IMT and s-ICAM-1 levels.

Prevalence of hypertension and prehypertension in individual dyslipidemic phenotypes

Fig. 1 shows the prevalence of hypertension, prehypertension, and normal BP in DLPs. The prevalence of prehypertension and of hypertension was significantly higher in all dyslipidemic patients (DLP 2, 3, and 4) com-
Prevalence (\%)

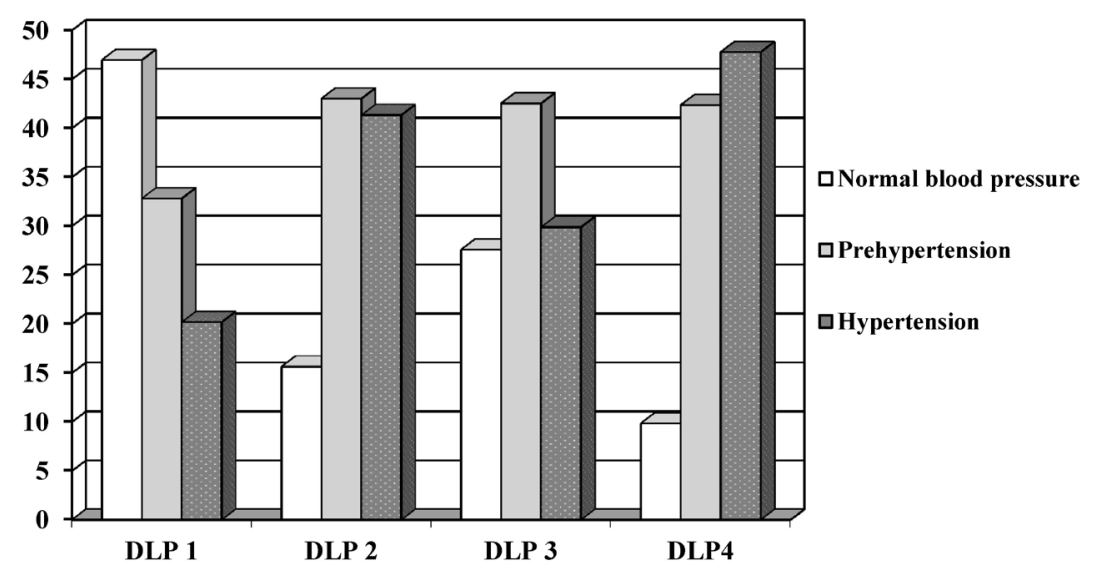

\begin{tabular}{|l|l|l|l|l|}
\hline & DLP 1 & DLP 2 & DLP 3 & DLP 4 \\
\hline \hline Prehypertension & $32,8 \%(65 / 198)^{\mathbf{b}, \mathbf{c}, \mathbf{d}}$ & $43,0 \%(77 / 179)^{\mathbf{a}}$ & $42,5 \%(37 / 87)^{\mathbf{a}}$ & $42,4 \%(86 / 203)^{\mathbf{a}}$ \\
\hline Hypertension & $20,2 \%(40 / 198)^{\mathbf{b}, \mathbf{d}}$ & $41,3 \%(74 / 179)^{\mathbf{a}, \mathbf{c}}$ & $29,9 \%(26 / 87)^{\mathbf{b , d}}$ & $47,8 \%(97 / 203)^{\mathbf{a}, \mathbf{c}}$ \\
\hline
\end{tabular}

Fig. 1. Prevalence of normal blood pressure, prehypertension and hypertension in individual dyslipidemic phenotypes.

DLP = dyslipidemic phenotype; DLP 1: apoB $<1.2 \mathrm{~g} / \mathrm{L}$ + TG $<1.5 \mathrm{mmol} / \mathrm{L}$, DLP 2: $\operatorname{apoB}<1.2 \mathrm{~g} / \mathrm{L}+\mathrm{TG} \geq$ $1.5 \mathrm{mmol} / \mathrm{L}$, DLP 3: apoB $\geq$ $1.2 \mathrm{~g} / \mathrm{L}+\mathrm{TG}<1.5 \mathrm{mmol} / \mathrm{L}$, DLP 4: $\operatorname{apoB} \geq 1.2 \mathrm{~g} / \mathrm{L}+\mathrm{TG}$ $\geq 1.5 \mathrm{mmol} / \mathrm{L}$.

Differences in hypertension and prehypertension between subgroups were analyzed with $\mathrm{X}^{2}$ test. Significant difference $P<0.05$ at least $-{ }^{a}$ vs. DLP

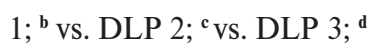
vs. DLP 4. 
Table 1. Basic characteristics in dyslipidemic phenotypes.

\begin{tabular}{|c|c|c|c|c|}
\hline & DLP 1 & DLP 2 & DLP 3 & DLP 4 \\
\hline $\mathrm{n} / \mathrm{men}$ & $198 / 67$ & $179 / 102$ & $87 / 36$ & $203 / 108$ \\
\hline Age (years) & $39.5 \pm 15.5^{\beta, \gamma, \delta}$ & $43.6 \pm 14.0^{a, \delta}$ & $45.1 \pm 14.4^{a}$ & $49.0 \pm 12.7^{\alpha, \beta}$ \\
\hline $\mathrm{TC}(\mathrm{mmol} / \mathrm{L})$ & $5.14 \pm 1.0^{\mathbf{b}, \mathbf{c}, \mathrm{d}}$ & $5.84 \pm 1.36^{\mathrm{a}, \mathbf{c}, \mathbf{d}}$ & $7.26 \pm 0.99^{\mathbf{a}, \mathbf{b}, \mathbf{d}}$ & $7.72 \pm 1.82^{\mathbf{a}, \mathbf{b}, \mathbf{c}}$ \\
\hline TG ${ }^{\mathrm{I}}(\mathrm{mmol} / \mathrm{L})$ & $1.0[0.77-1.2]^{\mathbf{b , d}}$ & $2.28[1.76-3.73]^{\mathrm{a}, \mathrm{c}}$ & $1.27[1.01-1.44]^{\mathbf{b , d}}$ & $2.57[2.01-3.76]^{\mathrm{a}, \mathrm{c}}$ \\
\hline HDL-C (mmol/L) & $1.67 \pm 0.42^{\mathrm{b}, \mathrm{d}}$ & $1.3 \pm 0.39^{\mathrm{a}, \mathrm{c}}$ & $1.64 \pm 0.42^{\mathrm{b}, \mathrm{d}}$ & $1.33 \pm 0.38^{\mathrm{a}, \mathrm{c}}$ \\
\hline LDL-C (mmol/L) & $3.02 \pm 0.84^{\mathrm{c}, \mathrm{d}}$ & $3.14 \pm 0.79^{\mathrm{c}, \mathrm{d}}$ & $5.04 \pm 1.01^{\mathrm{a}, \mathrm{b}}$ & $4.97 \pm 1.3^{\mathrm{a}, \mathrm{b}}$ \\
\hline non-HDL-C (mmol/L) & $3.48 \pm 0.88^{\mathbf{b}, \mathbf{c}, \mathbf{d}}$ & $4.55 \pm 1.34^{\mathrm{a}, \mathrm{c}, \mathrm{d}}$ & $5.63 \pm 1.01^{\mathrm{a}, \mathrm{b}, \mathrm{d}}$ & $6.39 \pm 1.81^{\mathrm{a}, \mathrm{b}, \mathrm{c}}$ \\
\hline apoB $(\mathrm{g} / \mathrm{L})$ & $0.87 \pm 0.19^{\mathrm{c}, \mathrm{d}}$ & $1.0 \pm 0.15^{\mathrm{c}, \mathrm{d}}$ & $1.39 \pm 0.19^{\mathbf{a}, \mathbf{b}}$ & $1.50 \pm 0.28^{\mathbf{a}, \mathbf{b}}$ \\
\hline apo A1 (g/L) & $1.66 \pm 0.33^{b, d}$ & $1.47 \pm 0.32^{\mathrm{a}}$ & $1.61 \pm 0.3$ & $1.52 \pm 0.33^{\mathrm{a}}$ \\
\hline apoB/apoA1 & $0.54 \pm 0.16^{\mathbf{b , c , d}}$ & $0.69 \pm 0.18^{\mathrm{a}, \mathbf{c}, \mathbf{d}}$ & $0.9 \pm 0.24^{\mathbf{a}, \mathbf{b}, \mathbf{d}}$ & $1.05 \pm 0.38^{\mathbf{a}, \mathbf{b}, \mathbf{c}}$ \\
\hline glycemia (mmol) & $4.86 \pm 0.56$ & $5.14 \pm 0.71$ & $4.92 \pm 0.65$ & $5.24 \pm 0.87$ \\
\hline Insulin ${ }^{\mathrm{I}}(\mathrm{mIU} / \mathrm{L})$ & $6.4[4.2-8.8]^{\mathrm{b,d}}$ & $8.8[6.2-12.7]^{\mathrm{a}, \mathrm{c}}$ & $6.3[3.9-8.5]^{\mathrm{b}, \mathrm{d}}$ & $8.4[5.9-11.5]^{\mathrm{a}, \mathrm{c}}$ \\
\hline $\mathrm{HOMA}^{\top}\left(\mathrm{mIU} * \mathrm{mmol} / \mathrm{L}^{2}\right)$ & $1.4[0.9-2.0]^{b, d}$ & $1.9[1.4-3.0]^{\mathrm{a}, \mathrm{c}}$ & $1.4[0.9-1.9]^{\mathbf{b , d}}$ & $1.8[1.3-2.8]^{\mathrm{a}, \mathrm{c}}$ \\
\hline C-peptide I (mg/L) & $1.8[1.4-2.4]^{\mathrm{b}, \mathrm{d}}$ & $2.5[1.9-3.3]^{\mathrm{a}, \mathrm{c}}$ & $1.7[1.4-2.4]^{\mathbf{b , d}}$ & $2.6[1.8-3.4]^{\mathrm{a}, \mathrm{c}}$ \\
\hline Proinsulin " (mIU/L) & $9.3[7.1-12.5]^{\mathrm{b}, \mathrm{d}}$ & $12.9[9.8-18.1]^{\mathrm{a}, \mathrm{c}}$ & $10.5[7.6-13.8]^{\mathrm{b}}$ & $12.0[9.4-17.6]^{\mathrm{a}}$ \\
\hline hs-CRP I (mg/L) & $1.0[0.5-2.3]^{\mathrm{d}}$ & $1.3[0.7-3.2]$ & $1.3[0.7-3.0]$ & $1.8[1.0-3.5]^{\mathrm{a}}$ \\
\hline $\mathrm{SBP}(\mathrm{mm} \mathrm{Hg})$ & $122.5 \pm 13.6^{\mathbf{b , d}}$ & $130.2 \pm 15.9^{\mathrm{a}}$ & $126.2 \pm 17.0$ & $131.7 \pm 14.7^{\mathrm{a}}$ \\
\hline DBP (mm Hg) & $75.8 \pm 9.2^{\mathbf{b}, \mathbf{d}}$ & $80.0 \pm 9.4^{\mathrm{a}}$ & $78.7 \pm 7.8$ & $81.7 \pm 8.0^{\mathrm{a}}$ \\
\hline $\operatorname{BMI}\left(\mathrm{kg} / \mathrm{m}^{2}\right)$ & $24.0 \pm 4.0^{\beta, \delta}$ & $27.5 \pm 6.1^{a, \gamma}$ & $24.8 \pm 2.9^{\beta, \delta}$ & $27.1 \pm 4.1^{a, y}$ \\
\hline Waist $(\mathrm{cm})$ & $79.8 \pm 11.7^{\mathrm{b}, \mathrm{d}}$ & $91.1 \pm 13.3^{\mathrm{a}, \mathrm{c}}$ & $83.3 \pm 9.9^{\mathbf{b}, \mathbf{d}}$ & $91.4 \pm 11.8^{\mathrm{a}, \mathrm{c}}$ \\
\hline MS ATP III (\%) & $3.0^{\mathbf{b}, \mathbf{d}}$ & $45.8^{\mathrm{a}, \mathrm{c}}$ & $2.3^{\mathbf{b , d}}$ & $49.3^{\mathrm{a}, \mathrm{c}}$ \\
\hline IMT (mm) & $0.59 \pm 0.12^{\mathrm{d}}$ & $0.66 \pm 0.14$ & $0.67 \pm 0.11$ & $0.72 \pm 0.14^{\mathrm{a}}$ \\
\hline $\operatorname{PAI}^{19}(\mathrm{ng} / \mathrm{ml})$ & $57.5[35.0-82.9]^{\mathbf{b , d}}$ & $74.7[46.4-99.4]^{\mathrm{a}}$ & $69.2[44.0-92.0]$ & $81.1[50.7-106.8]^{\mathrm{a}}$ \\
\hline s-ICAM-1 (ng/ml) & $490.1 \pm 143.2^{\mathrm{d}}$ & $521.4 \pm 213.3^{\mathrm{d}}$ & $482.1 \pm 148.3^{\mathrm{d}}$ & $567.1 \pm 202.0^{\mathbf{a}, \mathbf{b}, \mathbf{c}}$ \\
\hline
\end{tabular}

DLP = dyslipidemic phenotype; DLP 1: apoB $<1.2 \mathrm{~g} / \mathrm{L}+\mathrm{TG}<1.5 \mathrm{mmol} / \mathrm{L}$, DLP 2: apoB $<1.2 \mathrm{~g} / \mathrm{L}+\mathrm{TG} \geq 1.5 \mathrm{mmol} / \mathrm{L}$, DLP $3:$ apoB $\geq 1.2 \mathrm{~g} / \mathrm{L}$ $+\mathrm{TG}<1.5 \mathrm{mmol} / \mathrm{L}, \mathrm{DLP} 4:$ apoB $\geq 1.2 \mathrm{~g} / \mathrm{L}+\mathrm{TG} \geq 1.5 \mathrm{mmol} / \mathrm{L}$

$\mathrm{TC}=$ total cholesterol, TG $=$ triglycerides, HDL-C $=$ HDL cholesterol, LDL-C $=$ LDL cholesterol, non-HDL-C $=$ non-HDL cholesterol $(\mathrm{TC}-$ HDL-C), apoB = apolipoprotein B, apoA1 = apolipoprotein A 1, HOMA = homeostasis model assessment [fasting glycemia $(\mathrm{mmol} / \mathrm{L})$ fasting insulin $(\mathrm{mU} / \mathrm{L}) / 22.5$ ], hs-CRP $=$ high sensitivity $\mathrm{C}$ reactive protein, $\mathrm{SBP}=$ systolic blood pressure, $\mathrm{DBP}=$ diastolic blood pressure, $\mathrm{BMI}=$ body mass index, MS ATP III = metabolic syndrome diagnosed according to National Cholesterol Education Program Adult Panel III, IMT = intimamedia thickness, PAI-1 = plasminogen activator inhibitor-1, s-ICAM-1 = soluble intercellular cell adhesion molecule-1 Values are expressed as mean \pm S.D., for ${ }^{9}$ (parameters with skewed distribution) median [25 and 75 percentiles].

Differences in variables between subgroups were analyzed with ANOVA after adjustment for age, sex, BMI and smoking. Significant difference

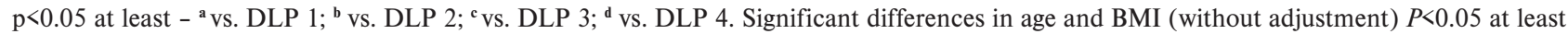
$-{ }^{a}$ vs. DLP $1 ;{ }^{\beta}$ vs. DLP $2 ;{ }^{\gamma}$ vs. DLP $3 ;{ }^{8}$ vs. DLP 4.

Parameters with skewed distribution (TG, proinsulin, insulin, C-peptide, HOMA, hs-CRP, PAI-1) were log transformed to normalize their distribution before statistical analysis. 
pared to normolipidmeic subjects (DLP 1). The odds ratio of the presence of prehypertension or hypertension in dyslipidemic subjects versus normolipidemic subjects are presented in Table 2. The cumulative prevalence of prehypertension/hypertension was also significantly higher particularly in hyper-TG DLPs (DLP 2 and 4). Subjects with DLP 1 had hypertension or prehypertension in $53.0 \%$ (105/198). Prehypertension or hypertension was found in $84.4 \%(151 / 179)$ of DLP 2 subjects and in $72.4 \%$ (63/87) of subjects with DLP 3. The highest prevalence of hypertension or prehypertension - 90.1\% (183/203) was found in DLP 4.

Relationship of blood pressure with other parameters

As a substantial percentage of hypertensive patients were being treated by anti-hypertensive drugs (see above), which influence the level of BP, only normotensive and prehypertensive subjects were enclosed in the subsequent analysis. SBP correlated with age $(\mathrm{r}=0.33 ; P<0.01)$, total cholesterol ( $\mathrm{r}=0.14 ; P<0.01)$, TG $(\mathrm{r}=0.16 ; P<0.01)$, non-HDL-C $(\mathrm{r}=0.13 ; P<0.01)$, fasting glycemia $(\mathrm{r}=0,14$, $P<0.01)$, C-peptide (r=0.10; $P<0.05)$, BMI ( $\mathrm{r}=0.23$;
$P<0.01)$ and waist ( $\mathrm{r}=0.32 ; P<0.01)$. By using multivariate backward stepwise regression analysis SBP was independently predicted by total cholesterol, non-HDL, waist circumference in all dyslipidemic subjects (DLP 2+3+4) and additionally by fasting glycemia in hypertriglyceridemic individuals (DLP 2+4) - see table 3. DBP correlated with age $(\mathrm{r}=0.25 ; P<0.01)$, total cholesterol $(\mathrm{r}=0.12 ; P<0.05)$, TG $(\mathrm{r}=0.13 ; P<0.01)$, non-HDL-C $(\mathrm{r}=0.12 ; P<0.05)$, apoB $(\mathrm{r}=0.16 ; P<0.01)$, HOMA (r=0.10; $P<0.05)$, BMI $(\mathrm{r}=0.21$; $P<0.01)$, waist $(\mathrm{r}=0.26 ; P<0.01)$ and PAI-1 $(\mathrm{r}=0.20$, $P<0.01$ ). By using backward stepwise regression analysis DBP was independently predicted by total cholesterol, non-HDL-C, waist circumference, and PAI-1 in hypertriglyceridemic subjects (DLP 2+4), in addition by apoB in subjects with DLP2, and only by waist circumference and PAI-1 in subjects with DLP 3 - see Table 3.

IMT according to blood pressure in individual dyslipidemic phenotypes

IMT rose from DLP 1 to DLP 4 (see Table 1). The highest elevation of IMT was observed in patients with simultaneously elevated levels of TG and of apoB (DLP

Table 2. Odds ratios ( $95 \%$ confidence interval) of presence of prehypertension or hypertension in dyslipidemic subjects (DLP 2,3,4) versus normolipidemic subjects (DLP 1).

\begin{tabular}{lll}
\hline & Prehypertension & Hypertension \\
\hline DLP 2 vs. DLP 1 & $1.545(1.016-2.348)^{*}$ & $2.784(1.763-4.397)^{* *}$ \\
DLP 3 vs. DLP 1 & $1.514(0.902-2.543)^{*}$ & $1.684(0.947-2.993)^{*}$ \\
DLP 4 vs. DLP 1 & $1.504(1.001-2.259)^{*}$ & $3.615(2.321-5.523)^{* * *}$ \\
\hline
\end{tabular}

DLP = dyslipidemic phenotype; DLP 1: apoB $<1.2 \mathrm{~g} / \mathrm{L}+\mathrm{TG}<1.5 \mathrm{mmol} / \mathrm{L}, \mathrm{DLP} 2:$ apoB $<1.2 \mathrm{~g} / \mathrm{L}+\mathrm{TG} \geq 1.5 \mathrm{mmol} / \mathrm{L}, \mathrm{DLP} 3:$ apoB $\geq 1.2 \mathrm{~g} / \mathrm{L}$ $+\mathrm{TG}<1.5 \mathrm{mmol} / \mathrm{L}$, DLP $4: \mathrm{apoB} \geq 1.2 \mathrm{~g} / \mathrm{L}+\mathrm{TG} \geq 1.5 \mathrm{mmol} / \mathrm{L}$.

${ }^{*} \sim P<0.05,{ }^{* *} \sim P<0.01,{ }^{* * *} \sim P<0.001 \ldots$ statistical significance level

Table 3. Predictive values of independent predictors on the levels of systolic and of diastolic blood pressure by multivariate backward stepwise regression analysis in dyslipidemic individuals.

\begin{tabular}{|c|c|c|c|c|c|c|}
\hline & \multicolumn{2}{|c|}{ DLP 2} & \multicolumn{2}{|c|}{ DLP 3} & \multicolumn{2}{|c|}{ DLP 4} \\
\hline & SBP & DBP & SBP & DBP & SBP & DBP \\
\hline \multirow{2}{*}{$\mathrm{TC}$} & $\beta=0.878$ & $\beta=0.522$ & $\beta=1.351$ & \multirow{2}{*}{ n.s. } & $\beta=0.854$ & $\beta=0.988$ \\
\hline & $P<0.001$ & $P<0.001$ & $P<0.001$ & & $P<0.001$ & $P<0.001$ \\
\hline \multirow{2}{*}{ non-HDL-C } & $\beta=-0.655$ & $\beta=-0.422$ & $\beta=-0.946$ & \multirow{2}{*}{ n.s. } & $\beta=-0.625$ & $\beta=-0.813$ \\
\hline & $P<0.001$ & $P<0.001$ & $P<0.001$ & & $P<0.001$ & $P<0.001$ \\
\hline apoB & - & $\begin{array}{l}\beta=0.269 \\
P<0.001\end{array}$ & - & n.s. & - & n.s. \\
\hline glycemia & $\begin{array}{l}\beta=0.242 \\
P<0.001\end{array}$ & - & n.s. & - & $\begin{array}{l}\beta=0.287 \\
P<0.001\end{array}$ & - \\
\hline \multirow{2}{*}{ waist } & $\beta=0.528$ & $\beta=0.368$ & $\beta=0.587$ & $\beta=0.580$ & $\beta=0.482$ & $\beta=0.498$ \\
\hline & $P<0.001$ & $P<0.001$ & $P<0.001$ & $P<0.001$ & $P<0.001$ & $P<0.001$ \\
\hline PAI-1 & - & $\begin{array}{l}\beta=0.259 \\
P<0.001\end{array}$ & - & $\begin{array}{l}\beta=0.416 \\
P<0.001\end{array}$ & - & $\begin{array}{l}\beta=0.322 \\
P<0.001\end{array}$ \\
\hline
\end{tabular}

$\mathrm{DLP}=$ dyslipidemic phenotype, $\mathrm{SBP}=$ systolic blood pressure, $\mathrm{DBP}=$ diastolic blood pressure, $\mathrm{TC}=$ total cholesterol, non-HDL-C $=$ non-HDL cholesterol (TC - HDL-C), apoB = apolipoprotein B, PAI-1 = plasminogen activator inhibitor-1, $\beta$ (beta) = standardized coefficient of regression, $P=$ statistical significance level 
IMT (mm)

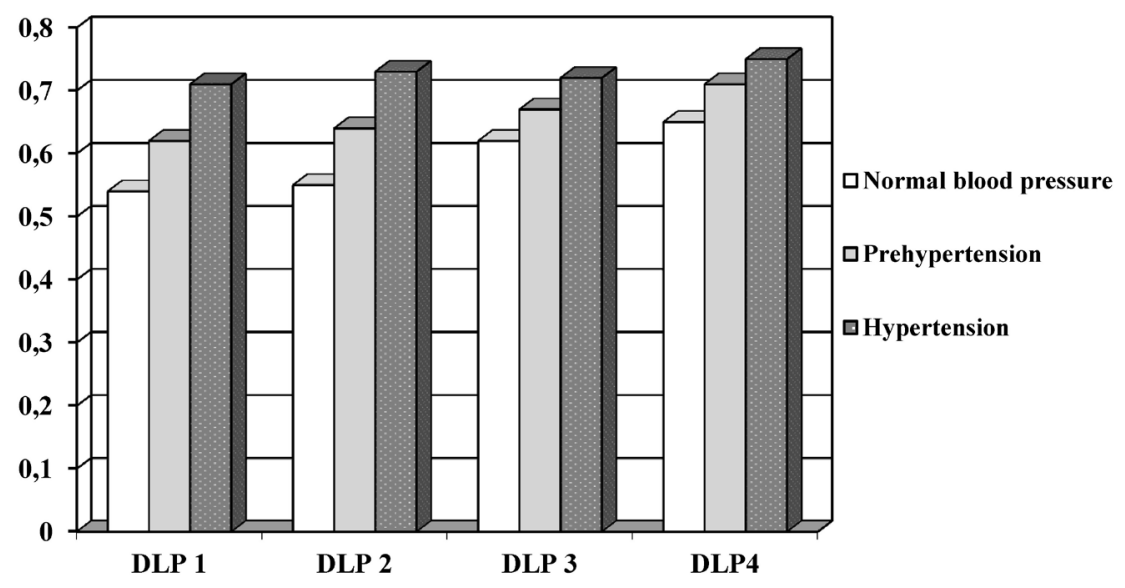

\begin{tabular}{|l|l|l|l|l|}
\hline & DLP 1 & DLP 2 & DLP 3 & DLP 4 \\
\hline IMT NBP $(\mathrm{mm})$ & $0.54 \pm 0.08^{\mathrm{c}, \mathrm{d}}$ & $0.55 \pm 0.07$ & $0.62 \pm 0.11^{\mathrm{a}}$ & $0.65 \pm 0.14^{\mathrm{a}}$ \\
\hline IMT PHN $(\mathrm{mm})$ & $0.62 \pm 0.12^{\mathrm{d}}$ & $0.64 \pm 0.13$ & $0.67 \pm 0.10$ & $0.71 \pm 0.15^{\mathrm{a}}$ \\
\hline IMT HN $(\mathrm{mm})$ & $0.71 \pm 0.14$ & $0.73 \pm 0.13$ & $0.72 \pm 0.10$ & $0.75 \pm 0.12$ \\
\hline
\end{tabular}

Fig. 2. Intima-media thickness according to blood pressure in individual dyslipidemic phenotypes.

DLP $=$ dyslipidemic phenotype; DLP 1: apoB $<1.2 \mathrm{~g} / \mathrm{L}$ + TG $<1.5 \mathrm{mmol} / \mathrm{L}$, DLP 2: apoB $<1.2 \mathrm{~g} / \mathrm{L}+\mathrm{TG} \geq$ $1.5 \mathrm{mmol} / \mathrm{L}$, DLP 3: apoB $\geq$ $1.2 \mathrm{~g} / \mathrm{L}+\mathrm{TG}<1.5 \mathrm{mmol} / \mathrm{L}$, DLP 4: apoB $\geq 1.2 \mathrm{~g} / \mathrm{L}+\mathrm{TG}$ $\geq 1.5 \mathrm{mmol} / \mathrm{L}$.

IMT $=$ intima-media thickness, NBP $=$ normal blood pressure, $\mathrm{PHN}=$ prehypertension, $\mathrm{HN}=$ hypertension Values are expressed as mean \pm S.D. Differences in IMT between subgroups were analyzed with ANOVA after adjustment for age, sex, BMI and smoking. Significant difference $P<0.05$ at least $-{ }^{a}$ vs. DLP 1 ; ${ }^{\mathrm{b}}$ Vs. DLP 2; ${ }^{\mathrm{c}}$ Vs. DLP 3; d vs. DLP 4.
4). In the whole group of all participants IMT was thicker in prehypertensive (IMT $=0.67 \pm 0.14 ; P<0.05)$ and in hypertensive (IMT $=0.73 \pm 0.12 ; P<0.05)$ patients respectively than in normotensive individuals (IMT $=0.57 \pm 0.10$ ) after adjustment for age, sex, BMI and smoking. Fig. 2 shows differences of IMT in various groups according to BP and the presence of various DLP. IMT of hypertensive and of prehypertensive subjects respectively was higher than of subjects with normal BP within all DLPs. By comparison between DLPs the significant differences were found between IMT of normotensive (DLP 1 versus DLP 3, and DLP 4 respectively) and prehypertensive subjects (DLP 1 versus DLP 4). Because anti-hypertensive drugs could influence BP and IMT, only untreated subjects were enrolled in the further analysis. IMT correlated with age $(\mathrm{r}=0.69$; $P<0.001)$, SBP ( $\mathrm{r}=0.35 ; P<0.001)$, DBP ( $\mathrm{r}=0.21 ; P<0.01)$, total cholesterol ( $\mathrm{r}=0.46 ; P<0.001)$, LDL-C ( $\mathrm{r}=0.39$; $P<0.001)$, non-HDL-C $(\mathrm{r}=0.45 ; P<0.001)$, TG $(\mathrm{r}=0.23$; $P<0.01)$, apoB (r=0.36; $P<0.001), \mathrm{C}$-peptide $(\mathrm{r}=0.15$; $P<0.01)$, BMI (r=0.23; $P<0.01)$ and waist $(\mathrm{r}=0.29 ; P<0.01)$. IMT correlated with 10 -year coronary heart disease risk in the whole group $(\mathrm{r}=0.48, P<0.001)$ and also in individual DLPs (DLP1 r=0.52; $P<0.001$, DLP2 r=0.47; $P<0.001$, DLP r $=0.38 ; P<0.01$, DLP r $=0.36 ; P<0.001)$. By using multivariate backward stepwise regression analysis IMT was independently predicted only by age (beta $=0.22 \pm 0.02$; $P<0.001$ ), total cholesterol (beta $=0.15 \pm 0.02 ; P<0.001$ ), SBP (beta $=0.38 \pm 0.05 ; P<0.001)$ and waist circumference (beta $=0.260 .05 ; P<0.001)$.

\section{DISCUSSION}

The results showed significantly higher prevalence of prehypertension/hypertension in clinically asymptomatic subjects with dyslipidemia (DLP 2, 3, and 4) than in normolipidemic individuals (DLP 1) even after adjustment for age, sex, smoking and body mass index. Cumulative prevalence of prehypertension or hypertension was the highest in the hypertriglyceridemic subjects (DLP 2 and 4). They had significantly increased blood pressure. SBP correlated with age, total cholesterol, TG, non-HDL-C, C-peptide, fasting glycemia, BMI and waist circumference. DBP correlated with age, total cholesterol, TG, non-HDLC, apoB, HOMA, BMI, waist circumference and PAI-1.

Many cross-sectional studies of different populations demonstrated that prehypertensive individuals had higher total cholesterol, LDL-C, TG and lower HDL-C than normotensive subjects ${ }^{6-8,20-30}$. Some prospective studies also showed that dyslipidemia could predict occurrence of incident hypertension by years ${ }^{31-33}$. The mechanism by which dyslipidemia leads to BP elevation is only partially understood. Dyslipidemia causes endothelial dysfunction, the loss of vasomotor reactivity and arterial stiffness ${ }^{34}$. We found total cholesterol, and non-HDL-C independent predictors for BP elevation. PAI-1 as a maker of endothelial dysfunction was independently associated with DBP in all dyslipidemic patients of this study, too. These findings may be explained by the suggesting pathogenetic mechanism. Lipid particles could activate recently identified liver $\mathrm{X}$ receptor, which is a potential regulator of renin expression $^{32,35}$. The activation of renin-angiotensin-aldosterone system (RAAS) increases BP and also leads (via angiotensin II) to PAI-1 expression ${ }^{36}$. PAI-1 can promote 
fibrosis, vascular remodeling, arterial stiffness and BP increase by preventing the activation of matrix metalloproteinases and the degradation of extracellular matrix by plasminogen activators and plasmin ${ }^{37}$. The significant increase of blood pressure and the highest cumulative prevalence of prehypertension/hypertension were detected in hypertriglyceridemic subjects and the levels of PAI-1 were significantly elevated in these patients too.

Hypertriglyceridemic subjects also showed increased markers of insulin resistance and of abdominal obesity. Many papers found the association between insulin resistance and prehypertension ${ }^{8,38,39}$ or hypertension ${ }^{40}$. Hyperinsulinemia directly increases sodium re-absorption in renal tubules and may lead to fluid retention ${ }^{41,42}$. Insulin activates sympathetic nervous system, increases catecholamine levels and the activity of RAAS (ref. ${ }^{43-45}$ ). However, not insulinemia alone but insulin resistance is usually related to blood pressure in prehypertensive and hypertensive patients and it is able to predict incident hyperten$\operatorname{sion}^{46-48}$. There was not found correlation between insulin levels and BP in the present study, too. Only C-peptide and fasting glycemia correlated with SBP, HOMA with DBP. However, in multivariate regression analysis no more specific marker of insulin resistance (except fasting glycemia) rested to predict BP levels. SDP and DBP $\mathrm{BP}$ were independently predicted by waist circumference. Abdominal obesity seems to play an independent role in $\mathrm{BP}$ elevation of all dyslipidemic patients. Among various anthropometric measurements waist circumference was detected the best predictor for incidence of hypertension ${ }^{49}$ and waist circumference was also independent predictor for progression of prehypertension to hypertension ${ }^{50}$. The possible mechanism linking abdominal obesity with BP elevation is production of adipokines. Visceral fat produces more angiotensinogen and PAI-1 than subcutaneous fat $^{51}$. The activation of RAAS leading to vasoconstriction and increase sodium re-absorption, together with chronic inflammation and endothelium dysfunction are probably the main causes of prehypertension and hypertension in patients with abdominal obesity.

The present study also showed increased IMT of prehypertensive, hypertensive patients respectively compared to normotensive individuals in all dyslipidemic phenotypes. IMT was independently predicted by age, total cholesterol, SBP, and waist circumference. It was closely related to 10-year coronary heart disease risk estimated using Framingham Risk Score ${ }^{15}$. Several papers also found IMT of the common carotid artery in patients with prehypertension or borderline hypertension thicker compared to normotensive controls ${ }^{52,53}$. Many studies detected the increase of IMT in patients with dyslipidemia. Our findings showed cumulative effect of dyslipidemia and BP increase on IMT. We found significant differences between IMT of prehypertensive subjects according to DLPs after adjustment for age, sex, smoking and BMI. Prehypertensive patients with DLP 4 had the same IMT as normolipidemic patients with hypertension. While the second group should be treated by antihypertensive drugs, the pharmacological treatment for prehypertension has been still recommended only in patients with diabetes mellitus, chronic kidney disease or coronary artery disease ${ }^{1}$. Also assessment of global cardiovascular risk in clinically asymptomatic dyslipidemic middle-aged patients with prehypertension only by SCORE(ref. ${ }^{54}$ ) or by Framingham Risk Score ${ }^{15}$ may not result to recommendation for hypolipidemic treatment. And thus measurement of IMT could constitute useful diagnostic tool for cardiovascular risk prediction and making decision on pharmacological treatment in these people. This noninvasive examination should be considered especially in asymptomatic dyslipidemic individuals with hyperapoB/ hyperTG phenotype, who have not reached the level of risk for initiation of therapy based on clinical and laboratory data only.

\section{CONCLUSION}

The prevalence of prehypertension was significantly higher in all dyslipidemic patients compared to normolipidemic subjects. Common prevalence of prehypertension and hypertension was the highest in the hypertriglyceridemic subjects. Prehypertensive and hypertensive patients had higher IMT than normotensive individuals in all dyslipidemic phenotypes. Dyslipidemia and blood pressure have cumulative effect on IMT. IMT measurement should be considered in asymptomatic individuals with hyperapoB/hyperTG phenotype to improve estimation of their cardiovascular risk.

\section{ABBREVIATIONS}

ANOVA, Analysis of variance; ACE-I, Angiotensinogen-converting enzyme inhibitors; ApoA1, Apolipoprotein A1; ApoB, Apolipoprotein B; ARBs, Angiotensin II receptor blockers; BBs, Beta blockers; BMI, Body mass index; BP, Blood pressure; CaCBs, Calcium channel blockers; CCA, Common carotid artery; CVD, Cardiovascular disease; DBP, Diastolic blood pressure; DLP, Dyslipidemic phenotype; ELISA, Enzyme-linked immunosorbent assay; HDL, High density lipoprotein; HDL-C, HDL cholesterol; HOMA, Homeostasis model assessment; Hs-CRP, High sensitivity $\mathrm{C}$ reactive protein; IMT, Intima-media thickness; IRMA, Immunoradiometric assay; IRLs, Imidazoline-1 receptor ligands; LDL, Low density lipoprotein; LDL-C, LDL-cholesterol; NHANES, National Health and Nutrition Examination Survey; NonHDL-C, Non-HDL cholesterol; PAI-1, Plasminogen activator inhibitor-1; RAAS, Renin-angiotensin-aldosterone system; RIA, Radioimmune assay; SBP, Systolic blood pressure; SPSS, Statistical package for the social sciences; S-ICAM-1, Soluble intercellular cell adhesion molecule-1; TC, Total cholesterol; TG, Triglycerides.

\section{ACKNOWLEDGEMENTS}

This work was supported by grant IGA MZCR NS/10284-3. 


\section{REFERENCES}

1. Chobanian AV, Bakris GL, Black HR, Cushman WC, Green LA, Izzo JL Jr, Jones DW, Materson BJ, Oparil S, Wright JT Jr, Roccella EJ; Joint National Committee on Prevention, Detection, Evaluation and Treatment of High Blood Pressure. National Heart, Lung, and Blood Institute; National High Blood Pressure Education Program Coordinating Committee. Seventh report of the Joint National Committee on Prevention, Detection, Evaluation, and Treatment of High Blood Pressure. Hypertension 2003;42:1206-52.

2. Julius S, Nesbitt SD, Egan BM, Weber MA, Michelson EL, Kaciroti N Black HR, Grimm RH Jr, Messerli FH, Oparil S, Schork MA; Trial of Preventing Hypertension (TROPHY) Study Investigators. Feasibility of treating prehypertension with angiotensin-receptor blocker. $\mathrm{N}$ Engl J Med 2006;354:1685-97.

3. Vasan RS, Larson MG, Leip EP, Evans JC, O'Donnell CJ, Kannel WB Levy D. Impact of high-normal blood pressure on the risk of cardiovascular disease. N Engl J Med 2001;345:1291-7.

4. Qureshi Al, Suri MF, Kirmani JF, Divani AA, Mohammad Y. Is prehypertension a risk factor for cardiovascular diseases? Stroke 2005;36:1859-63.

5. Mainous AG 3rd, Everett CJ, Liszka H, King DE, Egan BM Prehypertension and mortality in a nationally representative cohort. Am J Cardiol 2004;94:1496-500.

6. Greenlund KJ, Croft JB, Mensah GA. Prevalence of heart disease and stroke risk factors in persons with prehypertension in the United States, 1999-2000. Arch Intern Med 2004;164:2113-8.

7. Zhang Y, Lee ET, Devereux RB, Yeh J, Best LG, Fabsitz RR, Howard BV. Prehypertension, diabetes, and cardiovascular disease risk in a population-based sample: the Strong Heart Study. Hypertension 2006;47:410-4.

8. Cordero A, Laclaustra M, León M, Grima A, Casasnovas JA, Luengo E, del Rio A, Ferreira I, Alegria E. Prehypertension is associated with insulin resistance state and not with an initial renal function impairment. A Metabolic Syndrome in Active Subjects in Spain (MESYAS) Registry substudy. Am J Hypertens 2006;19:189-96.

9. Sniderman AD. Applying apoB to the diagnosis and therapy of the atherogenic dyslipoproteinemias: a clinical diagnostic algorithm. Curr Opin Lipidol 2004;15:433-8.

10. de Graaf J, Couture P, Sniderman A. A diagnostic algorithm for the atherogenic apolipoprotein B dyslipoproteinemias. Nat Clin Pract Endocrinol Metab 2008;4:608-18.

11. Campos H, Blijlevens E, McNamara JR, Ordovas JM, Posner BM, Wilson PW, Castelli WP, Schaefer EJ. LDL particle size distribution. Results from the Framingham Offspring Study. Arterioscler Thromb 1992;12:1410-9.

12. Demacker PN, Veerkamp MJ, Bredie SJ, Marcovina SM, de Graaf J, Stalenhoef AF. Comparison of the measurement of lipids and lipoproteins versus assay of apolipoprotein B for estimation of coronary heart disease risk: a study in familial combined hyperlipidemia. Atherosclerosis 2000;153:483-90.

13. Contois JH, McNamara JR, Lammi-Keefe CJ, Wilson PW, Massov T, Schaefer EJ. Reference intervals for plasma apolipoprotein B determined with a standardized commercial immunoturbidimetric assay: results from the Framingham Offspring Study. Clin Chem 1996;42:515-23.

14. Bachorik PS, Lovejoy KL, Carroll MD, Johnson CL. Apolipoprotein B and Al distributions in the United States, 1988-1991: results of the National Health and Nutrition Examination Survey III (NHANES III). Clin Chem 1997;43:2364-78.

15. National Cholesterol Education Program (NCEP) Expert Panel on Detection, Evaluation, and Treatment of High Blood Cholestero in Adults (Adult Treatment Panel III). Third Report of the National Cholesterol Education Program (NCEP) Expert Panel on Detection, Evaluation, and Treatment of High Blood Cholesterol in Adults (Adult Treatment Panel III) final report. Circulation 2002;106:3143-421.

16. National Cholesterol Education Program (U.S.) Risk assessment too for estimating your 10-year risk of having a heart attack [electronic resource], available from: http://hp2010.nhlbihin.net/atpiii/calculator.asp.

17. Matthews DR, Hosker JP, Rudenski AS, Naylor BA, Treacher DF, Turner RC. Homeostasis model assessment: insulin resistance and beta-cell function from fasting plasma glucose and insulin concentrations in man. Diabetologia 1985;28:412-9.
18. Karasek D, Vaverkova H, Frysak Z, Halenka M, Jackuliakova D, Novotny D, Lukes J. Soluble intercellular cell adhesion molecule-1 and vascular cell adhesion molecule-1 in asymptomatic dyslipidemic subjects. Int Angiol 2011;30:441-50.

19. Karasek D, Vaverkova $H$, Halenka $M$, Jackuliakova D, Frysak Z, Slavik L, Novotny D. Prothrombotic markers in asymptomatic dyslipidemic subjects. J Thromb Thrombolysis 2011;31:27-36.

20. Tsai PS, Ke TL, Huang CJ, Tsai JC, Chen PL, Wang SY, Shyu YK. Prevalence and determinants of prehypertension status in the Taiwanese general population. J Hypertens 2005;23:1355-60.

21. Grotto I, Grossman E, Huerta M, Sharabi Y. Prevalence of prehypertension and associated cardiovascular risk profiles among young Israeli adults. Hypertension 2006;48:254-9.

22. Lee JH, Hwang SY, Kim EJ, Kim MJ. Comparison of risk factors between prehypertension and hypertension in Korean male industrial workers. Public Health Nurs 2006;23:314-23.

23. Janghorbani $M$, Amini M, Gouya MM, Delavari A, Alikhani S, Mahdavi A. Nationwide survey of prevalence and risk factors of prehypertension and hypertension in Iranian adults. J Hypertens 2008;26:419-26.

24. Zhang M, Batu B, Tong W, Li H, Lin Z, Li Y, Zhang X, Zhang Y. Prehypertension and cardiovascular risk factor clustering among Mongolian population in rural and animal husbandry area, Inner Mongolia, China. Circ J 2009;73:1437-41.

25. Yu D, Huang J, Hu D, Chen J, Cao J, Li J, Gu D. Association between prehypertension and clustering of cardiovascular disease risk factors among Chinese adults. J Cardiovasc Pharmacol 2009;53:388-400.

26. Hageman PA, Pullen $\mathrm{CH}$, Walker SN, Boeckner LS. Blood pressure, fitness, and lipid profiles of rural women in the wellness for women project. Cardiopulm Phys Ther J 2010;21:27-34.

27. Sit JW, Sijian L, Wong EM, Yanling Z, Ziping W, Jianqiang J, Yanling C, WongTK. Prevalence and risk factors associated with prehypertension: identification of foci for primary prevention of hypertension. $J$ Cardiovasc Nurs 2010;25:461-9.

28. Ferguson TS, Younger N, Tulloch-Reid MK, Forrester TE, Cooper RS, Van den Broeck J, Wilks RJ. Prevalence of the metabolic syndrome in Jamaican adults and its relationship to income and education levels. West Indian Med J 2010;59:265-73.

29. Isezuo SA, Sabir AA, Ohwovorilole AE, Fasanmade OA. Prevalence, associated factors and relationship between prehypertension and hypertension: a study of two ethnic African populations in Northern Nigeria. J Hum Hypertens 2011;25:224-30.

30. Nery AB, Mesquita ET, Lugon JR, Kang HC, de Miranda VA, de Souza BG, AndradeJA, Rosa ML. Prehypertension and cardiovascular risk factors in adults enrolled in a primary care programme. Eur J Cardiovasc Prev Rehabil 2011;18:233-9.

31. Halperin RO, Sesso HD, Ma J, Buring JE, Stampfer MJ, Gaziano JM. Dyslipidemia and the risk of incident hypertension in men. Hypertension 2006;47:45-50.

32. Laaksonen DE, Niskanen L, Nyyssönen K, Lakka TA, Laukkanen JA Salonen JT. Dyslipidaemia as a predictor of hypertension in middleaged men. Eur Heart J 2008;29:2561-8.

33. Tohidi M, Hatami M, Hadaegh F, Azizi F. Triglycerides and triglycerides to high-density lipoprotein cholesterol ratio are strong predictors of incident hypertension in Middle Eastern women. J Hum Hypertens 2011; doi:10.1038/jhh.2011.70. [Epub ahead of print].

34. Oparil S, Zaman MA, Calhoun DA. Pathogenesis of hypertension. Ann Intern Med 2003;139:761-76.

35. Zelcer $\mathrm{N}$, Tontonoz $\mathrm{P}$. Liver $\mathrm{X}$ receptors as integrators of metabolic and inflammatory signaling. J Clin Invest 2006;116:607-14.

36. Feener EP, Northrup JM, Aiello LP, King GL. Angiotensin II induces plasminogen activator inhibitor-1 and -2 expression in vascular endothelial and smooth muscle cells. J Clin Invest 1995;95:1353-62.

37. Alexander CM, Werb Z. Proteinases and extracellular matrix remodeling. Curr Opin Cell Biol 1989;1:974-82.

38. Knobler $\mathrm{H}$, Abbasi F, Lamendola $\mathrm{C}$, Reaven $\mathrm{GM}$. Insulin resistance and cardiovascular disease risk factors in subjects with prehypertension. Diab Vasc Dis Res 2011;8:43-6.

39. Hwu CM, Liou TL, Hsiao LC, Lin MW. Prehypertension is associated with insulin resistance. QJM 2009;102:705-11.

40. Reaven GM. Relationships among insulin resistance, type 2 diabetes, essential hypertension, and cardiovascular disease: similarities and differences. J Clin Hypertens (Greenwich) 2011;13:238-43.

41. Skøtt P, Hother-Nielsen O, Bruun NE, Giese J, Nielsen MD, Beck- 
Nielsen $\mathrm{H}$, Parving HH. Effects of insulin on kidney function and sodium excretion in healthy subjects. Diabetologia 1989;32:694-9.

42. Endre T, Mattiasson I, Berglund G, Hulthén UL. Insulin and renal sodium retention in hypertension-prone men. Hypertension 1994;23:313-9.

43. Rooney DP, Edgar JD, Sheridan B, Atkinson AB, Bell PM. The effects of low dose insulin infusions on the renin angiotensin and sympathetic nervous systems in normal man. Eur J Clin Invest 1991;21:430-5.

44. Reaven GM, Lithell H, Landsberg L. Hypertension and associated metabolic abnormalities--the role of insulin resistance and the sympathoadrenal system. N Engl J Med 1996;334:374-81.

45. Kern W, Fittje A, Fohr W, Kerner W, Born J, Fehm HL. Increase in systolic blood pressure and catecholamine level during hyperinsulinemia in a placebo-controlled euglycemic clamp in healthy subjects. Exp Clin Endocrinol Diabetes 2000;108:498-505.

46. Saad MF, Rewers M, Selby J, Howard G, Jinagouda S, Fahmi S, Zaccaro D, Bergman RN, Savage PJ, Haffner SM. Insulin resistance and hypertension: the Insulin Resistance Atherosclerosis study. Hypertension 2004:43:1324-31.

47. Hwu CM, Liou TL, Hsiao LC, Lin MW. Prehypertension is associated with insulin resistance. QJM 2009;102:705-11.

48. Lai TS, Curhan GC, Forman JP. Insulin resistance and risk of incident hypertension among men. J Clin Hypertens (Greenwich) 2009;11:483-90.
49. Panagiotakos DB, Chrysohoou C, Pitsavos C, Skoumas J, Lentzas $Y$, Katinioti A, Stefanadis C. Hierarchical analysis of anthropometric indices in the prediction of 5-year incidence of hypertension in apparently healthy adults: the ATTICA study. Atherosclerosis 2009;206:314-20.

50. De Marco M, de Simone G, Roman MJ, Chinali M, Lee ET, Russell M, Howard BV, Devereux RB. Cardiovascular and metabolic predictors of progression of prehypertension into hypertension: the Strong Heart Study. Hypertension 2009;54:974-80.

51. Arner P. Regional differences in protein production by human adipose tissue. Biochem Soc Trans 2001;29:72-5.

52. Manios E, Tsivgoulis G, Koroboki E, Stamatelopoulos K, Papamichae C, Toumanidis S, Stamboulis E, Vemmos K, Zakopoulos N. Impact of prehypertension on common carotid artery intima-media thickness and left ventricular mass. Stroke 2009:40:1515-8.

53. Toikka JO, Laine H, Ahotupa M, Haapanen A, Viikari JS, Hartiala $\mathrm{JJ}$, Raitakari OT. Increased arterial intima-media thickness and in vivo $L D L$ oxidation in young men with borderline hypertension. Hypertension 2000;36:929-33.

54. Conroy RM, Pyörälä K, Fitzgerald AP, Sans S, Menotti A, De Backer G, DeBacquer $D$, Ducimetière $P$, Jousilahti $P$, Keil U, Njølstad I, Oganov RG, Thomsen T, Tunstall-Pedoe H, Tverdal A, Wedel H, Whincup P, Wilhelmsen L, Graham IM; SCORE project group. Estimation of tenyear risk of fatal cardiovascular disease in Europe: the SCORE project. Eur Heart J 2003;24:987-1003. 